\title{
Modelling of Magnetron Sputtering of Tungsten Oxide with Reactive Gas Pulsing
}

\author{
Tomáš Kubart,* Tomáš Polcar, Oliver Kappertz, Nuno Parreira, \\ Tomas Nyberg, Sören Berg, Albano Cavaleiro
}

Reactive sputtering is one of the most commonly employed processes for the deposition of thin films. However, the range of applications is limited by inherent instabilities, which necessitates the use of a complex feedback control of reactive gas (RG) partial pressure. Recently pulsing of the RG has been suggested as a possible alternative. In this report, the concept of periodically switching the RG flow between two different values is applied to the deposition of tungsten oxide. The trends in the measured time dependent RG pressure and discharge voltage are reproduced by a dynamical model developed for this process. Furthermore, the model predicts the compositional depth profile of the deposited film reasonably well, and in particular helps to understand the formation of the interfaces in the resulting multi-layer film.

\section{Introduction}

In reactive sputter deposition, it is frequently necessary to employ closed loop control of the reactive gas (RG) pressure to obtain high deposition rates and optimum film properties ${ }^{[1]}$ As a relatively simple alternative to this reactive deposition processes, modulated flow of the RG has been suggested and it has attracted considerable attention. More than 20 years ago, the basic idea was proposed by Aronson et al. ${ }^{[2]}$ and Thornton. ${ }^{[3]}$ They showed that by switching RG flow between two values it is possible to deposit compounds at higher deposition rates than could be achieved in compound mode.

Howson et al..$^{[4]}$ and Martin et al. ${ }^{[5]}$ used RG pulsing for the deposition of different oxides. Martin et al. ${ }^{[5]}$ reported a deposition rate for $\mathrm{TiO}_{2}$ as high as $70 \%$ of the metallic deposition rate. Billard and coworkers ${ }^{[6]}$ carried out extensive experiments on a similar technique, magnetron sputtering with low frequency modulation of the discharge current.

T. Kubart, O. Kappertz, T. Nyberg, S. Berg

The Ångström Laboratory, Uppsala University, Box 534, 75121 Uppsala, Sweden

Fax: (+46) 18 555095; E-mail: Tomas.Kubart@angstrom.uu.se

T. Polcar, N. Parreira, A. Cavaleiro

Mechanical Department, Polo Il of the Coimbra University, Pinhal

de Marrocos, 3030-788 Coimbra, Portugal
The fundamental idea of all the methods referred to above is similar - the periodic transition between metal and compound mode, i.e. between the high rate deposition of an oxygen-deficient layer and a subsequent oxidation at high oxygen partial pressure corresponding to low deposition rate.

Despite the successful use of this technique, understanding of its details remains poor. In particular, the observed minimum time for the transition between both modes could not be explained. One possible reason might be that previously published dynamical models neglected implantation of RG into the target surface, taking only chemisorption into account. ${ }^{[7,8]}$ However, as shown by Depla and De Gryse, ${ }^{[9]}$ the target surface is substantially modified by ion implantation and rather thick layers of compound are formed, ${ }^{[10]}$ which has to be taken into account when simulating the time response of the process. $^{[11]}$ We use a slightly modified version of the latter model to explain our experimental findings in the deposition of tungsten oxide with a modulated flow of RG.

\section{Experimental Part}

DC reactive magnetron sputtering experiments were performed using a Hartec semi-industrial deposition equipment, using a pure tungsten target $\left(150 \times 150 \mathrm{~mm}^{2}\right)$. Prior to deposition, the system was evacuated to a pressure better than $1 \times 10^{-3} \mathrm{~Pa}$ by a pump of $100 \mathrm{l} \cdot \mathrm{s}^{-1}$ nominal pumping speed. High purity argon was 
introduced at a constant flow of $12.5 \mathrm{sccm}$ corresponding to $0.2 \mathrm{~Pa}$. The RG, high purity oxygen, was injected in rectangular pulses of duration $t_{\text {on }}$ and with a period $T$. During $t_{\text {on }}$ the flow was $q_{\mathrm{H}}=$ $20 \mathrm{sccm}$, for the rest of the period $q_{\mathrm{L}}=0 \mathrm{sccm}$. Throughout the whole experiment the discharge current was kept constant at $6 \mathrm{~mA} \cdot \mathrm{cm}^{-2}$. The tungsten oxide coatings were deposited onto heat-treated tool steel (AISI M2), glass and (111) silicon wafers which were cleaned by ion gun sputtering prior to deposition. The target to substrate distance was $65 \mathrm{~mm}$, and a substrate bias of $-70 \mathrm{~V}$ was applied. No substrate rotation was used and the deposition was performed without external heating, leading to substrate temperatures lower than $350^{\circ} \mathrm{C}$. The thickness of the films was evaluated by step profilometry, and the deposition rates were calculated from the film thickness and the sputtering time. The composition depth profile of the coatings was measured by $\mathrm{X}$-ray photoelectron spectroscopy (XPS).

\section{Model}

The dynamic model used in this report is an extension of our previous work, which is based on the concept introduced by Berg et al. ${ }^{[12,13]}$ In the basic model, the formation of compound was attributed to chemisorption only, while recent research shows the importance of ion implantation. ${ }^{[9,10]}$ To account for this, two different implantation mechanisms are included. ${ }^{[11]}$ First, the implantation that occurs as a result of ionized RG colliding with the target and, second, the implantation that occurs when ions collide with the target surface and knock-in the chemisorbed RG at the surface.

The subsurface region is divided into several layers to describe the compositional depth profile of the target caused by implantation effects. It is assumed that all the sputtered particles are neutral atoms. The model is one-dimensional, i.e. a uniform distribution of the ion current density over the target erosion area and deposition flux over the substrate surface is assumed. This simplification significantly reduces the computational time, while for steady-state the shape of hysteresis curves remains unchanged,${ }^{[13]}$ and the influence on transitions is supposed to be of second order only.

Both total pressure and discharge voltage are recorded during the sputtering experiments and need to be described by the model. Changes of the former correspond to variations in oxygen partial pressure for constant argon flow and pumping speed. The voltage provides indirect information on the present state of the target, but was not included in our previous models. The extension we suggest here is similar to an approach proposed by Buyle et al. ${ }^{[14]}$ He attributed changes in the target voltage mainly to the variation of the secondary electron (SE) coefficient which in turn affects the voltage required to sustain the discharge. The discharge voltage is assumed to be indirectly proportional to the $\gamma_{\mathrm{SE}}$, total SE yield of the target surface.

There are two basic mechanisms influencing the SE yield. The value of SE yield is different for metallic and compounded surface, which causes the different voltages in the compound and metal mode. Often the value of secondary electron yield is supposed to be higher for oxides than for pure metals due to longer electron mean free path in the insulating materials. However, many exceptions from that rule exist, where the voltage in compound mode is higher than in metal. ${ }^{[15]}$ When operating close to the transition between both cases, a different effect becomes significant. At the target surface, a layer of chemisorbed oxygen may exist. Such a layer would decrease the SE yield $\mathrm{d}^{[16]}$ and therefore cause an increase in voltage.

In our model, the voltage is calculated in the following way. Four different values of SE yield are assumed according to the composition of the target's two topmost layers. The total (effective) SE yield of the target is directly proportional to the contribution of each target area

$$
\gamma_{\mathrm{SE}}=\theta_{\mathrm{mm}} \gamma_{\mathrm{mm}}+\theta_{\mathrm{cc}} \gamma_{\mathrm{cc}}+\theta_{\mathrm{cm}} \gamma_{\mathrm{cm}}+\theta_{\mathrm{mc}} \gamma_{\mathrm{mc}}
$$

where the subscripts $\mathrm{mm}, \mathrm{cc}, \mathrm{cm}$ and $\mathrm{mc}$ stand for metal on metal, compound on compound, etc. fraction of the target surface, and $\gamma_{\mathrm{ii}}$ is the SE yield of the corresponding part of the target.

When the target is completely metallic or compounded, the voltage is dominated by $\gamma_{\mathrm{mm}}$ and $\gamma_{\mathrm{cc}}$, the SE yield of clean metal and compound, respectively. In the transition region, two more parameters become important. A single layer of compound on a metal is assumed to correspond to a layer of oxygen at the metallic surface, which leads to a decrease in SE yield. A thin surface layer of metal covering compound is assumed not to influence the SE yield substantially, $\gamma_{\mathrm{mc}}$ therefore equals to $\gamma_{\mathrm{cc}}{ }^{[17]}$ The fractions of $\gamma_{\mathrm{cm}}$ and $\gamma_{\mathrm{mc}}$ increase during transition from one operating point to another, while in the steady-state their influence is less pronounced.

The depth profile of the deposited coating is calculated in a straightforward manner. The actual deposition rate is determined from the flux of sputtered metal atoms, and the composition is determined by the substrate coverage $\theta_{\mathrm{s}}$. The film thickness is then obtained by integrating the deposition rate over time.

\section{Results and Discussion}

\section{Steady-State Hysteresis}

In a first step, steady-state hysteresis curves of oxygen pressure and discharge voltage versus RG flow were recorded. The parameters necessary to describe system geometry and material properties have been extracted by fitting, since for oxides only insufficient data on effective SE and sputtering yields is available. Two different target materials, tungsten and titanium, were used to ensure better reliability of the fitting procedure and to separate system geometry from material properties. Experimental and simulated curves for tungsten oxide are shown in Figure 1; the parameters used in the simulations are listed in the Appendix. A small shift of the simulated transition region towards lower flows can be attributed to the simplifications used in the model.

\section{RG Pulsing}

Process curves for pulsing of RG with a period of $T=50 \mathrm{~s}$ and pulse length of $t_{\text {on }}=25 \mathrm{~s}$ are shown in Figure 2. There 
a)

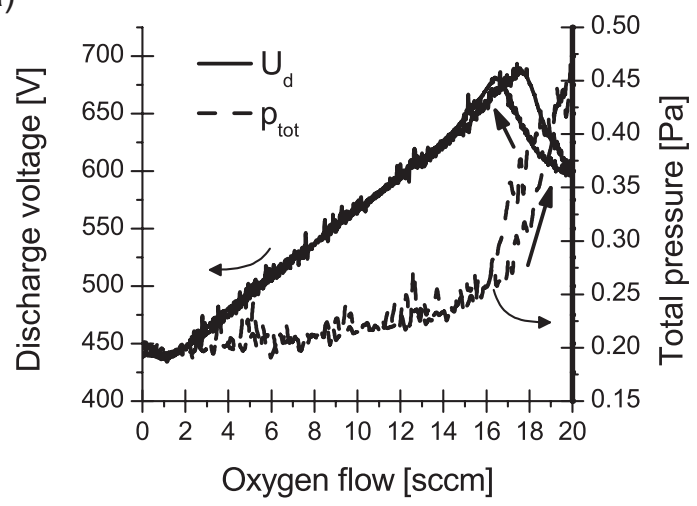

b)

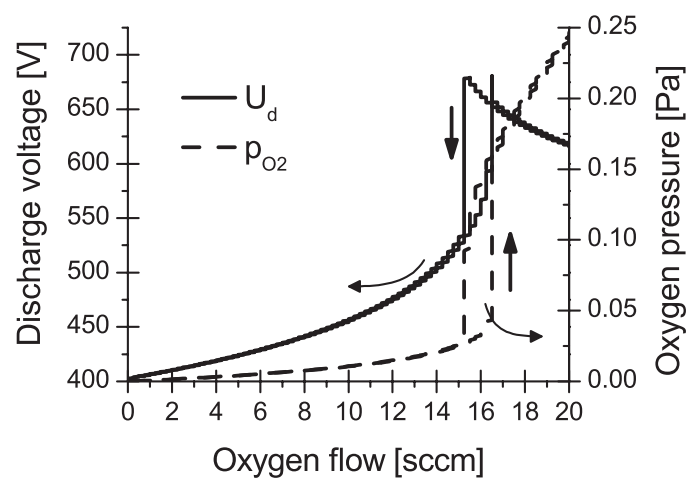

Figure 1. Hysteresis curves of DC reactive sputtering of W-O measured by experiment (a) and simulation (b).

is satisfactory agreement between experimental (a) and simulated (b) curves. Note that while the RG was pulsed between $q_{\mathrm{L}}=0$ and $q_{\mathrm{H}}=20 \mathrm{sccm}$ in the experiment, in the simulations $q_{\mathrm{H}}=18 \mathrm{sccm}$ was taken because of the shift mentioned in the previous paragraph. As discussed in ref. ${ }^{[11]}$ the time the process requires to change from one mode to another depends strongly on the new value of RG flow. This effect is pronounced when the flow is close to the critical flow as shown later.

Four different regimes can be distinguished on the process curves. ${ }^{[5]}$ Two of them correspond to steady-state processing points. For low RG flow $q_{\mathrm{RG}}=q_{\mathrm{L}}$, the sputtering is performed in metal mode and the pressure and voltage correspond to their steady-state values. The same holds for high RG flow $q_{\mathrm{RG}}=q_{\mathrm{H}}$ when the process is in compound mode. After abruptly increasing the RG flow from $q_{\mathrm{L}}$ to $q_{\mathrm{H}}$ the metal-to-compound transition occurs. During this transition, the target surface is converted from metal to compound, and a subsurface compound layer is formed by implantation of RG. The main factor influencing the transition time is, however, the presence of pure metal on the substrate and wall surfaces. Those surfaces represent substantial gettering capacity and sufficient RG has to be introduced to convert them into compounds. Then, at higher RG partial pressure, poisoning of the target may further proceed. When the RG is switched from $q_{\mathrm{H}}$ to $q_{\mathrm{L}}$, the opposite transition takes place. During a compoundto-metal transition, a built-up layer of compound from the target surface has to be removed. Since the sputtering yield for compounds, especially oxides, is much lower than for metals, the time for altering the target surface is longer in this direction. The main process parameter affecting the time scale for this process is the current density at the target. However, in this case, the effect of RG gettering at the chamber walls, which dominates the metal-tocompound transition, can be neglected. As a result, the total transition time in Figure 2 is longer for metalto-compound changeover.

An interesting feature of the discharge voltage is the appearance of peaks during transitions (Figure 2), which are reproduced by our simulations. These peaks correspond to an increase in $\theta_{\mathrm{cm}}$ the simulated fraction of the target surface with one thin layer of compound covering the target metal. This supports the validity of De Gryse's and our initial assumption. Behaviour of the RG pressure is discussed in more details in the study. ${ }^{[1]}$

The composition of the deposited films is also strongly affected by these transitions. XPS depth profiles (Figure 3) and high resolution SEM micrographs ${ }^{[18]}$ of films deposited with parameters corresponding to Figure 2 reveal a layered a)

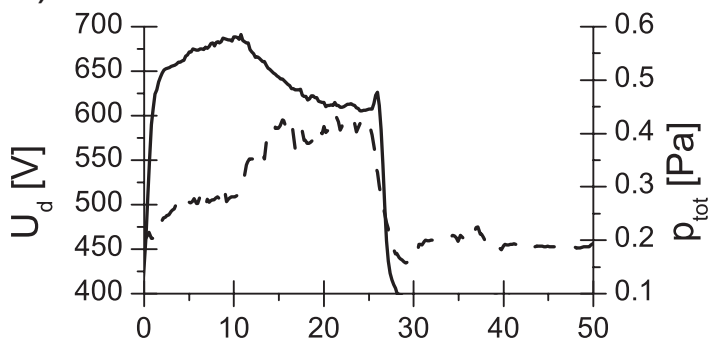

b)

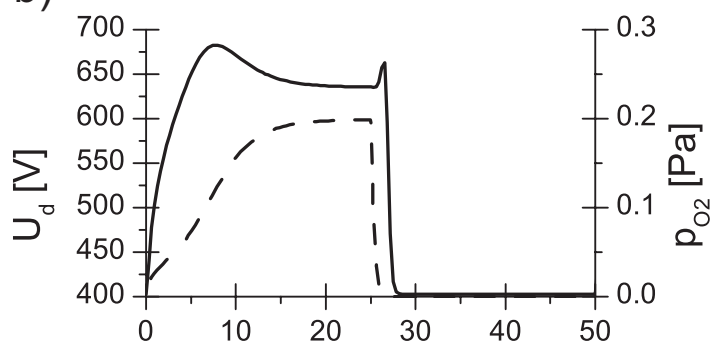

Figure 2. Experimental (a) and simulated (b) process parameters during the pulsing of RG. Process curves for discharge voltage (solid) and pressure (dashed) are shown for $T=50 \mathrm{~s}$ and $t_{\mathrm{on}}=25 \mathrm{~s}$. 
a)

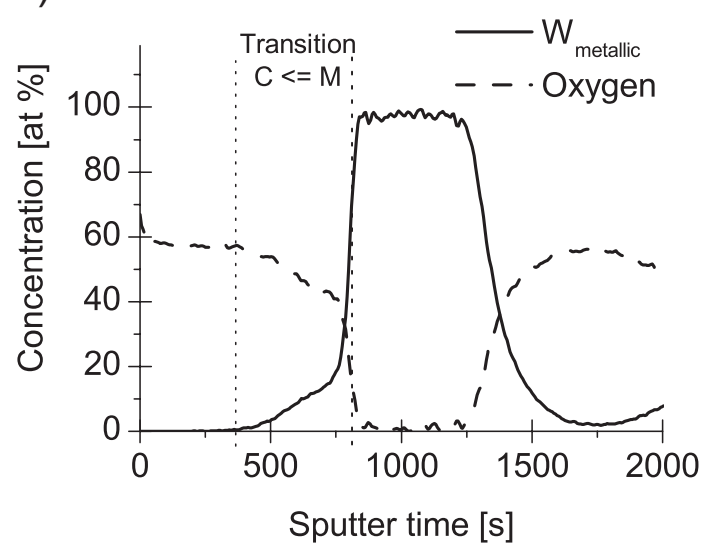

b)

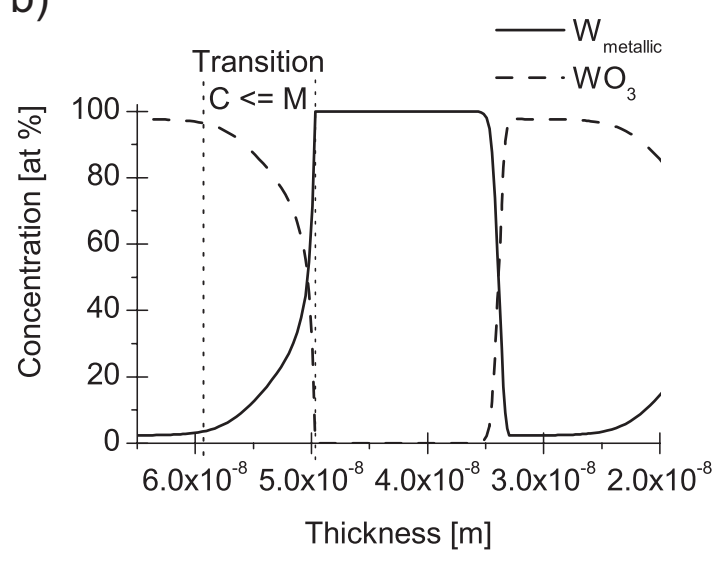

Figure 3. Experimental XPS (a) and simulated (b) depth profiles of coatings deposited for $T=50 \mathrm{~s}$ and $t_{\text {on }}=25 \mathrm{~s}$. Transitions in film composition are apparent when switching from metal to compound mode. In both graphs the surface is to the left.

structure of the coatings. Again the experiment (Figure 3a) and simulations (Figure $3 \mathrm{~b}$ ) are in a good agreement. At the interfaces between the metal and the compound, substoichiometric regions can be identified, which are deposited during process transitions. In our case, the layered structure is rather pronounced due to the relatively high deposition rate of more than $2 \mathrm{~nm} \cdot \mathrm{s}^{-1}$, which prevents oxidation of the already deposited film by diffusion. ${ }^{[19]}$

Since the pulsing parameters influence the stoichiometry, deposition rate and thickness of each single layer and define the interfaces, they can be tuned according to desired properties of the deposited coating. A prolonged transition time is beneficial to obtain higher deposition rates. Generally, with increasing oxygen flow, the mass deposition rate initially increases. This effect is caused by incorporation of oxygen into the film, and therefore total

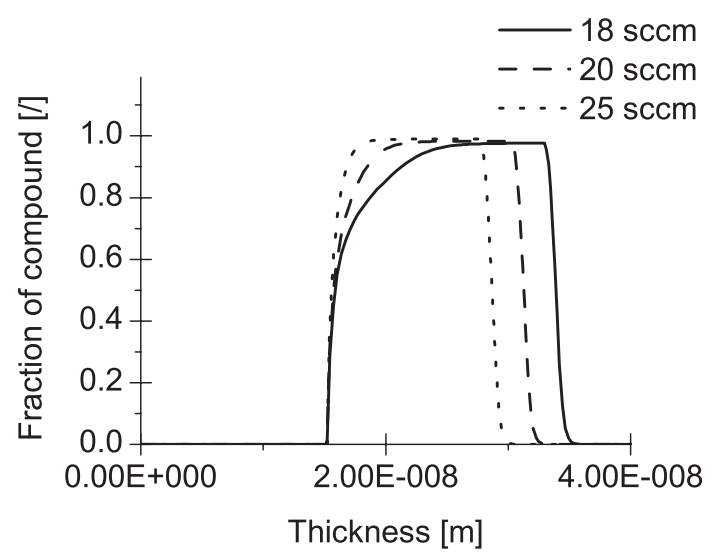

Figure 4. Predicted composition (fraction of compound) of deposited layers for different values of upper flow $q_{\mathrm{H}}$. Other parameters were kept constant: $T=50 \mathrm{~s}, t_{\mathrm{on}}=25 \mathrm{~s}, q_{\mathrm{L}}=0 \mathrm{sccm}$. The higher the value of $q_{\mathrm{H}}$ the sharper is the interface due to the reduced transition time. mass deposition rate increases even though the flux of metal atoms decreases slightly. Since the density of tungsten oxide is substantially lower than the density of pure tungsten, the deposition rate (thickness/time) is even more amplified in the case of tungsten oxide. Consequently, a prolonged dwell in the transition mode leads to higher deposition rate. A drawback of sputtering in the transition region is the potential presence of substoichiometric regions in the coating.

When sharper interfaces between metal and compound layer are required, transition times must be reduced. The simplest way to do so is to increase the upper flow of RG $q_{\mathrm{H}}$. The influence of this quantity is shown in Figure 4. The gettering capacity of metallic surfaces is constant, and therefore the necessary amount of RG is reached faster at higher flows resulting in a sharper interface. A similar approach can be used for the compound-to-metal transition. For sharp interfaces it is necessary to switch RG flow completely off, while a proper reduction of flow allows reaching higher deposition rate. For the interface sharpness the limiting factor is the time necessary to clean the target.

\section{Conclusion}

A dynamical model describing the reactive sputtering process with pulsing of the RG has been developed. It is possible to simulate various process parameters such as RG pressure, discharge voltage and composition of deposited film. This model has been applied to the reactive deposition of tungsten oxide with a satisfactory agreement between simulations and experimental results.

It is possible to relate the discharge voltage to the composition of the sputtering target and hence to the composition of the coating. This information may be 
helpful when a feedback control based on discharge voltage is used. Our results also support the validity of the two layered model for describing the discharge voltage.

The response times in pulsed reactive process are closely related to different parameters of the pulses, mainly upper and lower values of the mass flow. When sharp interfaces are required, it is necessary to reduce the transition time by switching between operating points sufficiently far away from the hysteresis region. By changing the shape of the pulses it is possible to deposit multilayers with tailormade compositional interfaces between metal and compound layers.

Acknowledgements: This work was supported by the Swedish Foundation for Strategic Research (SSF) Strategic Research Center in Materials Science for Nanoscale Surface Engineering $\left(\mathrm{MS}^{2} \mathrm{E}\right)$ and by the European Union through the NMP3-CT-2003-505948 project "HARDECOAT". The authors gratefully acknowledge Dr. Nuno Carvalho for SEM/EDX studies. This project was supported by Volkswagenstiftung, "Fundação para a Ciência e a Tecnologia" of Portugal for the financial support through the POCI/ V.5/A0034/2005 project "HARDECOAT - extension", and the SFRH/BD/16528/2004 Ph.D. Scholarship.

Received: September 11, 2006; Revised: October 16, 2006; Accepted: November 17, 2006; DOI: 10.1002/ppap.200731301

Keywords: dynamic modelling; gas pulsing; reactive sputtering; tungsten oxide

\section{Appendix}

The following parameters have been used for simulations: Target area $A_{\mathrm{t}}=0.008 \mathrm{~m}^{2}$, substrate area $A_{\mathrm{s}}=0.2 \mathrm{~m}^{2}$, thickness of implanted layer $N=16$ atomic layers is uniformly divided into 16 slabs, sticking coefficient of the RG on the target surface $\alpha_{\mathrm{tm}}=0.05$, on the substrate $\alpha_{\mathrm{sm}}=0.1$, sticking coefficient of atomic oxygen $\alpha_{\mathrm{ms}}=1$, sputtering yield of metal from the metallic surface $Y_{\mathrm{mm}}=1.1$, sputtering yield of metal from the compound
$Y_{\mathrm{mc}}=0.10$, sputtering yield of the RG atoms $Y_{\mathrm{cc}}=0.3$, forward sp. yield $Y_{\mathrm{kcc}}=0.6$, stoichiometry of the compound $x_{\mathrm{c}}=3, n_{\mathrm{s}}=1.623 \mathrm{E}+19 \mathrm{~m}^{2}, T=300 \mathrm{~K}$, discharge current $J=1.35$ A, pumping speed $S_{\mathrm{p}}=0.100 \mathrm{~m}^{3} / \mathrm{s}$, chamber volume $V=64 \mathrm{l}$, ionization efficiency $\eta_{\mathrm{Ar}}=1, \eta_{\mathrm{RG}}=0.2$, argon pressure $p_{\mathrm{Ar}}=0.2 \mathrm{~Pa}$, atoms per RG molecule $k_{\mathrm{RG}}=2$. Secondary electron yields: $\gamma_{\mathrm{mm}}=0.10, \gamma_{\mathrm{cc}}=0.082, \gamma_{\mathrm{cm}}=$ 0.0205, $\gamma_{\mathrm{mm}}=0.082$, Density of the metal $\rho_{\mathrm{m}}=$ $19250 \mathrm{~kg} \cdot \mathrm{m}^{-3}$ and for oxide $\rho_{\mathrm{c}}=7200 \mathrm{~kg} \cdot \mathrm{m}^{-3}$.

[1] W. D. Sproul, D. J. Christie, D. C. Carter, Thin Solid Films 2005, 491, 1.

[2] A. J. Aronson, D. Chen, W. H. Class, Thin Solid Films 1980, 72, 535.

[3] J. A. Thornton, Thin Solid Films 1981, 80, 1.

[4] R. P. Howson, N. Danson, I. Safi, Thin Solid Films 1999, 351, 32.

[5] N. Martin, A. R. Bally, P. Hones, R. Sanjines, F. Levy, Thin Solid Films 2000, 377, 550.

[6] D. Mercs, F. Lapostolle, F. Perry, A. Billard, C. Frantz, Surf. Coat. Technol. 1999, 119, 916.

[7] E. Kusano, J. Appl. Phys. 1993, 73, 8565.

[8] L. B. Jonsson, T. Nyberg, S. Berg, J. Vac. Sci. Technol. A 2000, 18, 503.

[9] D. Depla, R. De Gryse, Surf. Coat. Technol. 2004, 183, 184.

[10] Y. Abe, K. Takamura, M. Kawamura, K. Sasaki, J. Vac. Sci. Technol. A 2005, 23, 1371.

[11] T. Kubart, O. Kappertz, T. Nyberg, S. Berg, Thin Solid Films 2006, 515, 421.

[12] S. Berg, H. O. Blom, T. Larsson, C. Nender, J. Vac. Sci. Technol. A 1987, 5, 202

[13] S. Berg, T. Nyberg, Thin Solid Films 2005, 476, 215.

[14] G. Buyle, W. De Bosscher, D. Depla, K. Eufinger, J. Haemers, R. De Gryse, Vacuum 2003, 70, 29.

[15] O. Kappertz, Ph.D. Thesis, RWTH Aachen, Aachen 2004; http://darwin.bth.rwth-aachen.de/opus/volltexte/2004/800/.

[16] R. De Gryse, D. Depla, J. Haemers, Proceedings of 48 th Annual SVC Technical Conference Denver, Colorado, April 2005, p. 290.

[17] D. Depla, H. Tomaszewski, G. Buyle, R. De Gryse, Surf. Coat. Technol. 2006, 201, 848.

[18] N. M. G. Parreira, T. Polcar, A. Cavaleiro, Surf. Coat. Technol. 2007, 201, 5481.

[19] N. M. G. Parreira, N. J. M. Carvalho, A. Cavaleiro, Thin Solid Films 2006, 510, 191. 